\title{
The Most Controversial Subject in Dentistry
}

\author{
Gene McCoy* \\ Dentist in San Francisco, California, USA
}

*Corresponding author: Gene McCoy, Dentist in San Francisco, California, USA, E-mail: genemccoydds@sbcglobal.net

Received: 25 Aug, 2021 | Accepted: 14 Sep, 2021 | Published: 20 Sep, 2021

Citation: McCoy G (2021) The Most Controversial Subject in Dentistry. Int J Dent Oral Health 7(7): dx.doi.org/10.16966/2378-7090.382

Copyright: (c) 2021 McCoy G. This is an open-access article distributed under the terms of the Creative Commons Attribution License, which permits unrestricted use, distribution, and reproduction in any medium, provided the original author and source are credited.

\section{Introduction}

Dental occlusion had always been defined as teeth in closure [1], but at one point, there was a movement within the dental profession to modify the meaning. The dentists behind the movement, Mohl explained, felt that the definition: "Did not truly define the field because its' significance was much more than the occlusal contact relationship of the dentition" [2]. They felt that the word occlusion should be construed as an understanding of the masticatory system itself. In due course the movement was successful, and the word occlusion became synonymous with the masticatory system, but without rules. There was no explanation as to what constituted an ideal occlusion or how to achieve it, so practitioners were unsure of their goals. The lack of clarity subsequently prompted practitioners to formulate their own ideas as to how the system should work which made it impossible to develop intelligent diagnostic criteria and promulgate treatment.

This commentary/opinion is an account of the factors that have made this subject the most controversial subject in dentistry, and suggestions for the restorative dentist to achieve and maintain a healthy, comfortable, and efficient masticatory system.

\section{Exactly, what is occlusion?}

This is the heart of the problem, an understanding of just what occlusion signifies. In medicine, it means to close, obstruct, or prevent a passage such as blood in an artery or vein [3]. In dentistry however, it is understood to be the manner in which teeth come together in closure [4]. Seems simple enough, but over time the dental definition was modified to include the contact of teeth during those excursive movements of the mandible essential to mastication [5]. This was curious since it is not normal to eat our food in excursive movements and recognizing that teeth rarely touch when mastication does occur. Still, another interpretation was imminent.

Up to a point, all of the dental dictionaries had defined dental occlusion as the closure of teeth except one: Jablonski's Dictionary of Dentistry [6]. Dentists were becoming comfortable with using the word occlusion for just about anything related to the masticatory system which instigated a movement to personalize the definition and Jablonski gave them that opportunity.
Stanley Jablonski was a prolific writer of medical and dental books including a Russian-English dictionary. He used an encyclopedic approach, and to explain areas of particular complexity, he relied on glossaries and consultants as primary sources of information. To compile his Dictionary on Dentistry he used 31 consultants, 13 of which were dentists and 18 credentialed authorities in dental academia [6].

It was from their contribution of information, ideas, and opinions that Jablonski formulated his definition of occlusion and then the consultants turned around and quoted him as provenance.

\section{Jablonski's definition of occlusion}

"The relationship between all the components of the masticatory system in normal function, dysfunction, and parafunction, including the morphological and functional features of contacting surfaces of opposing teeth and restorations, occlusal trauma and dysfunction, neuromuscular physiology, the temporomandibular joint and muscle function, swallowing and mastication, psychophysiological status, and the diagnosis, prevention, and treatment of functional disorders of the masticatory system [6]."

Despite the good intentions, empowering the word occlusion to describe the masticatory system was just that: a description, not an analysis. It did not explain how the system should work which created a distraction rather than an adjunct when evaluating the system itself and things got complicated. Dentists knew how to analyze the status of a patient's masticatory system, but they did not understand occlusion's new significance. What did it mean exactly? What were the guidelines? How does one assign a meaning to a word that has two definitions?

There were no directives. Questions began to emerge along with multiple concepts that described six different mandibular positions, 16 different mandibular movements, and thirty different occlusal relationships [4]. The American Dental Association (ADA) needed clarity and wanted to resolve these issues. In 1952, the ADA sponsored a Nomenclature Conference to discuss and understand these different points of view [7]. The official purpose was to interpret the general concept of occlusion in its' broadest significance. The chairman was George B. Denton and there were 30 participants. There was so much dissention regarding the format that the conference considered 
only the concepts of occlusion rather than the terms which stood for the concepts. Both stationary and moving phenomena were to be considered. Each presented concept was judged to be either; "harmonious medium occlusion" (functional) or "disharmonious peripheral occlusion” (parafunctional).

The conference ended without resolving any of the disagreements, no official terminology was adopted, and there were no authoritative conclusions. It is interesting to note that in this attempt to understand the essence of occlusion, two categories were recognized; function and parafunction. The profession continued to debate the controversies.

In 1975 another attempt was made to resolve these controversies and establish credible guidelines for practitioners to follow regarding occlusion. The American Association of Dental Schools (AADS) initiated a workshop in New Orleans to investigate and survey the trends and goals of occlusal education [8]. Sponsoring institutions were Louisiana State University, the University of California, San Francisco, and the University of Southern California. The original workshop committee, within the Dental Anatomy and Occlusion Section of the AADS, consisted of: Dr. James Butler, chair, Dr. Rex Ingram, co-chair, Dr. Marwan Abou-Rass, editor, Dr. Ben Pavone, Dr. William Solberg, and Dr. Howard Bruggers. Additional consultants were: Dr. E.E. Jeansonne, Dr. Carl Rieder, Dr. Richard Blagbrough, Dr. Daniel Isaacson, and Dr James Bruch.

The major goal of the workshop was to prepare a manual to serve as an educational model for the planning and presentation of an occlusion curriculum, for undergraduates. The premise was to explain every aspect of the curriculum so that students could have a clear objective on what end goals to strive for with good, simple, and logical points. However, there was no explanation as to what these end goals were, nor was there an explanation as to what courses were qualified to achieve them. In addition, there was no formulation of a model of excellence in regard to the performance of the masticatory system: how it works efficiently and how to make corrections when it does not. However, two interesting points came to light. Dr. Parker Mahan, University of Florida, stated that a preferred goal was: "to understand normal stomatognathic system function, to recognize occlusal dysfunction, to determine its' etiology, and how to treat it." This observation mirrored the only distinction regarding occlusion in the 1952 Nomenclature Conference, i.e.; harmonious medium occlusion (functional) $v s$ disharmonious peripheral occlusion (parafunctional).

The other interesting point was presented by one of the speakers, Dr. Willy G. Krogh-Poulsen, Royal Dental College, Copenhagen; "I think that the term occlusion should be restricted to define only contact relations of the teeth, and that a new term should be created which will encompass the broader meaning of occlusion." He suggested that the term Physiodontics might be considered to serve as an appropriate term. He went on to say; "A deep understanding of the form-function of the relationships must be achieved or the student will not understand the needs of prevention against dysfunction and their sequelae." The participants in this workshop were also asked to define an optimal design for the relationship between the occlusion curriculum and other dental disciplines:this request was not fulfilled. In spite of the fact that the workshop failed to establish precise guidelines for practitioners to follow, the ADA Council on Dental Education initiated separate departments dedicated to occlusion (1980) in 51\% of the existing dental schools [9]. They were not successful.

In 1983, a new report; Special Guidelines in Occlusion, was developed by AADS members Dr. James W. Buckman and Dr. Jeffery
P. Okeson [10]. Although this report, using the 1975 workshop as a reference, was entitled Special Guidelines, it failed to define them.

Curiously, the credibility of this report was undermined by the following statement: "Although these guidelines were recommended as course development aids and suggested as official AADS policy, there are not official policy statements of the AADS and should not be construed as recommendations for restrictive requirements." There was no explanation as to the reason why. What is the current situation?

Understanding occlusion, 2013, is an update which begins with, "Frustration and confusion still reign over this complicated topic" [11]. In this article, author Jackie Syrop reviews differing philosophies of occlusion and interviews occlusion experts to gain insight about the current state of occlusion with the disappointing comment that there is a "willingness among dentists to concede that no single philosophy works for all cases."

Among the experts interviewed was John Kois, DMD, MSD, director of the Kois Center in Seattle, Washington who stated that; "Occlusion is the new frontier that is virtually untapped by most of the profession" [11]. But wait a minute; this is not a new conversation. Kois' statement seems to imply that something is missing: that maybe, we could have been doing something better? A frontier indicates that there is a limit to our present state of knowledge and there are new things to be learned, but there is not a single aspect of the masticatory system that we haven't analyzed, pondered, debated, and discussed thoroughly. We just were victims of entrenched thinking. We just need to rethink what we do know.

\section{Discussion}

The morphing of the word occlusion should never have happened. It was a major distraction to our understanding of the masticatory system: how it functions properly and what we need to do when it is in a state of parafunction. In linguistics, it is not unusual for the meaning of a word to change. It is called a semantic shift and it happens for various reasons. There can be a broadening where the word becomes more inclusive than an earlier meaning, or there can be a narrowing where the meaning becomes less inclusive, however this was different. A description of the dentition in closure was changed to a neuromuscular description of the masticatory system as a whole. This is called a polysemy, where the new interpretation becomes radically different from the original usage. It was a deliberate distortion to suit an agenda and with sincere respect, it did not make sense. The masticatory system has three main components: the occlusion, the muscles of mastication, and the condyle (Figure 1). If we now refer to the masticatory system as the occlusion system or the occlusion curriculum (Figure 2), what do we call the contact of teeth in closure?

A biological system is dependent on each component part to contribute to its' efficient function as a whole. If we rename the system to one of its' component parts, occlusion, how do we differentiate between the closure of teeth and the system? Occlusion is not a curriculum nor is it a system, but rather a component part of an existing system.

The human body has 12 major body systems. A biological system is a group of organs that are specifically designed to work together to perform a certain task. The health care practitioner who specializes in the care of any particular system needs to understand four simple truths; how the system functions efficiently and in good health, the component parts that enable that system to do so, how to identify the signs and symptoms of disease or parafunction, and how to correct or manage the problem. As the cardiologist is responsible for the 


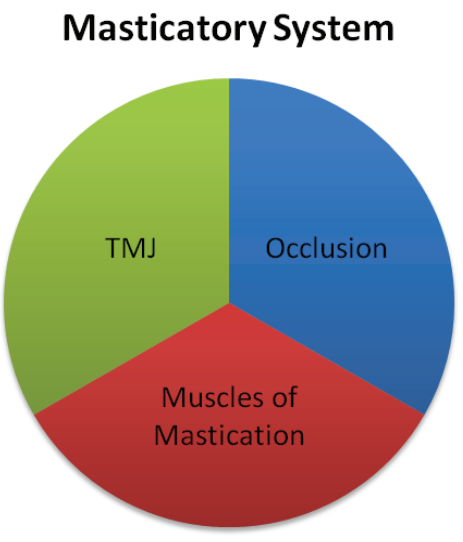

Figure 1: The Masticatory system is composed of three components.

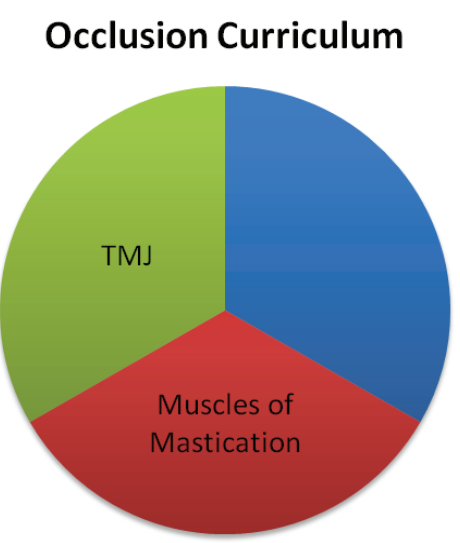

Figure 2: The Occlusion curriculum does not detail the teeth in closure.

cardiovascular system, so is the dentist for the masticatory system which is actually a minor system, the gateway to the larger digestive system whose sole purpose is to convert food in the alimentary canal into an absorbable form. The masticatory systems' job is to break down pieces of food to begin that process. It accomplishes this task with two opposing, u-shaped rows of overlapping teeth that interact with each other. When a patient presents for dental restoration, the practitioner focuses on two objectives: the project at hand and the status of the masticatory system. If the patient's system is functioning comfortably and efficiently, the dentist then concentrates on the immediate project whether it is an alloy restoration, crown, or removable prosthetics. If however, the system is affected with parafunction, this must be addressed before the restorative project. The polysemy diverted practitioner's attention from the masticatory system to an undefinable goal.

So, if our goal is an ideally functioning masticatory system with ideal dental occlusion in an ideal world, how would one describe it? Dawson opines; "We are dealing with a beautifully designed system that functions in perfect harmony as long as all its structural components are correctly inter-related" [12]. He's talking about the masticatory system, not occlusion. He then asks; "What is the specific relationship of teeth closure to functional harmony and stability of the system?" [12].

Now he's referring to occlusion. So, let's try to answer Dawson's question.
Defining an ideally functioning masticatory system: No matter the number, arrangement of teeth, or structural differences between the mandible and the maxilla, an ideally functioning masticatory system would be one that is devoid of parafunction. Although the mandible has the flexibility of lateral movement, all functional movements such as eating, talking, or swallowing are vertical. In the interim of normal function, the mandible is at rest.

Defining ideal occlusion: What is the most ideal relationship that teeth should touch each other in closure that ensures an ideally functioning masticatory system? With the exception of anomalies, our biological architects designed all our body parts to be perfect. Our dentition is no different. It was designed to cut through food and to harmoniously interact with the muscles of mastication and the TMJ. Figure 3 demonstrates nature's original design [13].

There are two salient observations: first, the contact is limited to the occluding cusp which directs force vectors along the longitudinal axis to be distributed circumferentially via the roots to the supporting alveolar bone and second, the incline planes have no contact at all creating a generousintra-incline space to hold, cut, and diminish food particles as they begin their journey through the digestive system. This space also not only reduces collisions during mastication but creates an anterior-posterior resistance free zone to accommodate changing mandibular positions during postural changes. In addition, condylar repositioning is ensured upon swallowing. Although our biological architects have demonstrated a perfect example of occlusal contact for harmonious function of the masticatory system, we are not to conclude that all dentitions should be equilibrated to obtain or maintain this objective. For many, the loss of the original sharp architecture of the occlusal surfaces, for whatever reason, may be a natural phenomena, and does not automatically indicate that an equilibration is required. The key is comfort. There are also the architectural differences in our skeletons due to genetics. Since not everyone has a class I jaw relationship and a perfect alignment of teeth, nature's example demonstrates that, when considering an equilibration, vertical loading is preferred and heavy lateral contacts are not.

\section{What is the relationship between occlusion and TMJD?}

This is another legacy of the polysemy. The reason this question is so difficult to answer is that, it's not the appropriate question. We should be asking if the TMJ is uncomfortable because the masticatory system is affected with parafunction and then determine if the way the teeth coming together is a contributing factor. It would be difficult to do a study to determine a credible relationship between parafunction and TMJD, as there are just too many variables such as the type, strength, and frequency of the parafunction and the biological strength of the patient. There are patients who have ground their teeth down to the gingiva with no discomfort to the TMJ, and those who have developed TMJD after the delivery of one uncomfortable restoration that initiated parafunction.

In my 50 years of restorative dentistry, I have not seen a single TMJD patient that did not exhibit signs of dental compression syndrome (DCS), so whatever the etiology of the TMJD, since clenching applies pressure to the TMJ, it is good common sense to focus on reduction and management of the same.

\section{Summary}

It is the obligation of every restorative dentist to promote oral health and to prevent disease.

To promote oral health, dentists must maintain good systemic physiology. Physiology, from the Latin meaning "The study of nature," 


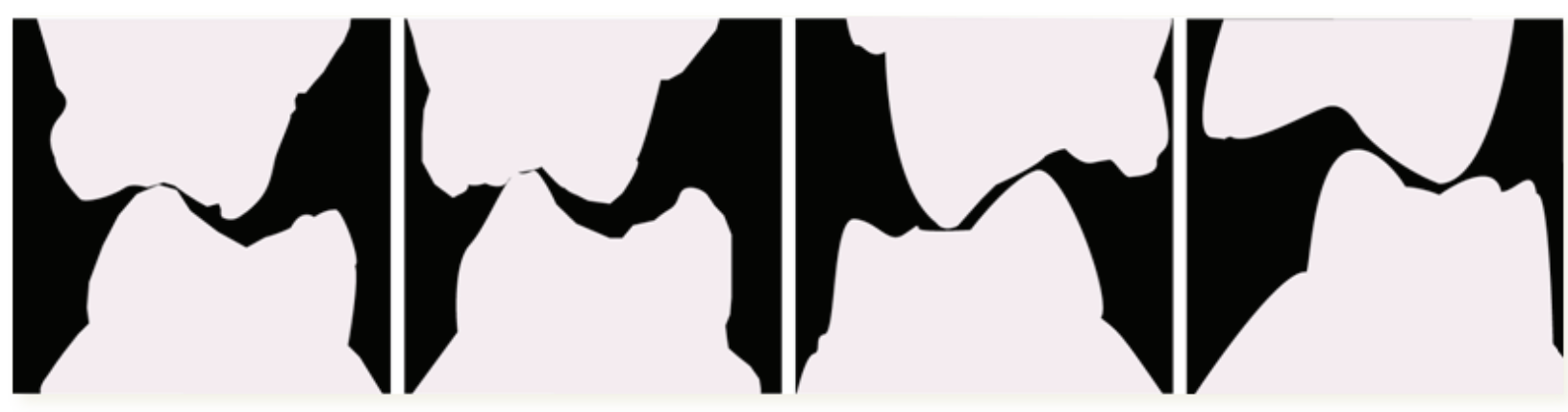

Figure 3: Unworn molars in occlusion.

is an understanding of various body parts and their functions. Aristotle was the first to describe how form and function govern anatomical studies. The masticatory system is characterized by three main components (the TMJ, the muscles of mastication, and the design of the dentition) that interact to form a coherent whole. It should be the mandate of every restorative dentist to understand and maintain the harmonious relationship of these three components to promote the oral health of the system.

The most damaging disease to threaten the integrity of any masticatory system is parafunction. It should also be the mandate of every restorative dentist to recognize its' signs and symptoms and to council patients as to it's' etiology and management.

\section{References}

1. Dorland WAN (1898) Dorland's Pocket Medical Dictionary. $1^{\text {st }}$ Edition, W.B. Saunders Company. Philadelphia.

2. Mohl NDA (1988) Textbook of Occlusion. Quintessence Publishing Co., Inc. Chicago, Illinois, USA.

3. Taber CW (1960) Taber's Cyclopedic Medical Dictionary. Eighth Edition, F.A. Davis Company, Philadelphia.
4. Zwemer TJ (1998) Mosby's Dental Dictionary. $1^{\text {st }}$ Edition. Mosby, Inc., St. Louis, Missouri.

5. Dorland WAN (1959) Dorland's Medical Dictionary. 20 th Edition. W.B. Saunders Company.

6. Jablonski S (1998) Jablonski's Dictionary of Dentistry. $2^{\text {nd }}$ Edition. Krieger Publishing Company, Malabar, Florida.

7. American Dental Association (1953).

8. Proceedings of the workshop on Occlusal Education (1975).

9. Curricular Guidelines in Occlusion (1983) J Dent Educ 47: 561-565.

10. Syrop J (2013) Understanding Occlusion. Inside Dentistry 9: 46-58.

11. Dawson P.E. The Concept of Complete Dentistry. A workbook, copyright 1990 \& 1994.

12. McCoy G (2020) The Great Occlusion Fiasco. J Oral Implantrol 46: 139-144.

13. McCoy G (1999) Dental Compression Syndrome, A New Look at an Old Disease. J Oral Implantrol 5:35-49. 